\title{
ON THE ROLE OF DIFFERENT LYMPHOCYTE SUBPOPULATIONS IN THE COURSE OF CORONAVIRUS MHV IV (JHM)-INDUCED ENCEPHALITIS IN LEWIS RATS
}

\author{
S. Schwender, A. Hein, H. Imrich, and R. Dörries \\ Institut für Virologie und Immunbiologie der Universität Würzburg \\ Versbacher Str. 7 \\ 8700 Würzburg
}

\section{INTRODUCTION}

The neurotropic strain of the mouse hepatitis virus (MHV), MHV-IV or JHM virus, causes neurological disorders in rats and mice when given intracerebrally 1,2 . Depending on the rat strain and the age of the infected animal different courses of disease have been observed. Up to 2 weeks post partum Lewis (LEW) rats develop an acute fatal encephalitis, whereas animals older than 3 weeks usually do not succumb to the infection 3 but suffer from a subacute paralytic disease accompanied by multiple neurological disorders. However, the majority of them recovers completely roughly 3 weeks past infection 4 . Since rats are expected to be immunologically competent at the age of 3 weeks, it is conceivable that maturity of the immune system probably plays a major role in the clinical course of the infection. We have recently shown, that $\mathrm{T}$ - and B-lymphocytes infiltrate into the central nervous system of these rats 4 and that the humoral immune response most likely contributes significantly to convalescence 5 .

Here, we report on the clinical course of the intracerebral infection with JHM virus in LEW rats after immunosuppression and reconstitution with defined lymphocyte subsets prior to infection. The data add further support to the idea, that the virus-specific antibody response is essential for a mild or subclinical course of the infection. 


\section{MATERIALS AND METHODS}

Animals. Lewis (LEW) rats were obtained from "Zentralinstitut für Versuchstierzucht", Hannover, Germany, and were kept under sterile conditions. They were infected intracerebrally with $10^{3}$ PFU of JHM virus. The animals were observed daily for neurological symptoms, that were scored according to the following scale: (0) no clinical sings; (1) uncommon social behaviour, loss of tail tone; (2) paresis of 1 or 2 legs, nervous behaviour; (3) paresis of more than 2 legs or paralysis of one or two legs, body trembling; (4) tetraplegia, or restless running and jumping, reaching sometimes a (5) moribund state. 4 (mod.)

Immunosuppression. LEW rats were whole body irradiated with $6(0) \mathrm{rad} \gamma$ of a ${ }^{137 \mathrm{Cs}}$ source. Immunosuppression was controlled by intraperitoneal immunization of irradiated rats with keyhole limpet hemocyanine $(\mathrm{KLH})$ and a subsequent assay of lymphocytes from spleen and lymph nodes for their capacity to secrete antigen-specific antibodies on a single cell basis using an ELISpot assay 5. In addition, an ELISA was performed by standard procedures to determine KLH-specific antibodies in serum specimens.

Preparation of lymphocyte subsets and reconstitution of animals. Lymphocytes for reconstitution of irradiated animals were isolated from spleen, cervical lymph nodes (LN) and mesenterial LN of uninfected syngeneic age-matched animals and transferred through the lateral tail vein within a few hours after irradiation. Compositions of transferred cells were as follows (table 1, columns \#1-\#8): non-separated lymphocytes (\#8), pure Tlymphocyte subsets (CD4+-, \#3; CD8+ T-cells, \#4), both T-cell subsets in the absence of B-lymphocytes (\#5) and different combinations of T-cell subsets and B-lymphocytes (\#6\#8). Each animal received that amount of purified cells, corresponding to their proportion in $2 \times 10^{8}$ unseparated, naive lymphocytes; i.e. they received $1.5 \times 108$ cells if, for instance, $75 \%$ of the cells in question were positive for that specific marker in the unseparated population.

Purification of lymphoid cell populations was performed by immunomagnetic procedures using the appropriate panel of monoclonal antibodies. The resulting viable cells were quantitated by trypan blue exclusion and quality of the purification was checked in a Becton \& Dickinson FACScan flow-cytometer using FITC-labeled antibodies 4. Routinely, less than $1 \%$ contamination was obtained after two depletion cycles.

Titration of virus. Brain and spinal cord (CNS) were taken from sacrificed animals at day 9 past infection. The weight of each CNS was determined and the tissue was homogenized in a $5 \mathrm{ml}$ Dounce homogenizer. The resulting suspension was clarified by centrifugation, the supernatant serially diluted and assayed for infectious virus in triplicates on a microtiter plate using DBT-cells as targets 6 . The TCID 50 was regarded as 
the highest dilution were DBT-monolayers in at least 2 of 3 wells developed more than $50 \%$ cytopathic effect.

\section{RESULTS AND DISCUSSION}

To dissect the role of individual lymphocyte subsets in the course of JHM virusinduced encephalitis in rats, animals were immunosuppressed and reconstituted with defined lymphocyte populations prior to infection. Immunosuppressive treatment was controlled in irradiated and KLH-immunized animals. They were assayed for their proliferative T-lymphocyte response to the mitogen Con $\mathrm{A}$ and their capability to mount a KLH-specific antibody response. After whole body irradiation only $0.2 \%$ of $3 \mathrm{H}$-thymidine was incorporated into Con-A stimulated lymph node cells compared to lymphocytes from untreated rats. The IgM response reached only $0.02 \%$ of the level obtained in normal rats and an IgG response is no longer detectable as determined by an ELISpot assay as well as by a KLH-specific ELISA. These data indicate, that the whole body irradiation of rats with $600 \mathrm{rad} \gamma$ is sufficient to completely suppress an antigen-specific immune reaction up to day 9 pi.

After infection development of neurological symptoms was recorded up to 9 dpi. The data are summarized in table 1 . Non-irradiated, infected rats developed clinical signs at day 8 pi with increasing severity on the next day (table 1, \#1). More or less the same course of clinical signs was observed in irradiated animals to which $2 \times 10^{8}$ non-separated lymphocytes were transferred. In both assays less than $500 \mathrm{TCID}_{50} / \mathrm{g}$ CNS virus were recovered, showing that the intravenous reconstitution of the immune system with lymphocytes was successful (table 1, \#8), and indicating the tight control of the infection by an intact immune system. In irradiated but not reconstituted animals incidence and severity of disease was comparable to the latter animals, with a remarkable difference with respect to the type of neurological disease (table $1, \# 2$ ). In the course of the infection most of these animals reacted by a highly nervous attitude of movement, pointing to a spread of the virus to grey matter rather than limitation to the white matter as usually seen in the subacute courses of the infection. The presence of JHM virus at $8000 \mathrm{TCID}_{50} / \mathrm{g}$ CNS in these animals clearly disclosed the lack of virus neutralization and thus supports the idea of viral spread to the cerebral cortex, brain stem and spinal cord. Similar results were reported from JHM virus-infected mice, where irradiation or cyclophosphamide-induced immunosuppression leads to a predominant gray matter manifestation instead of demyelinating disease 7 .

More severe courses of the JHM infection and an earlier onset of disease compared to irradiated but fully reconstituted or not reconstituted rats were observed in those animals which received either CD4+ T-cells, CD8+ T-cells or both together (table 1, \#3, \#4 and \#5, 
resp.). In combination with high titers of infectious JHM virus in the CNS of these animals, three important conclusions can be drawn from these findings: (1) The virus itself undisturbed by an immune response is rather slow in developing clinical signs of the infection, although after 9 dpi strong neurological symptoms can be seen (data not shown), (2) T-lymphocytes in the absence of B-lymphocytes amplify and accelerate severity and development of disease and (3) despite T-lymphocytes react to virus-infected cells in the brain tissue they are not able to significantly reduce infectious virus. Again these results are in line with findings of Fleming et al., 7 who described in the mouse model a correlation between development of disease and the presence of T-lymphocytes in the transferred lymphocyte population. In this context, it is important to note that in our rat model not only CD8+ T-lymphocytes or a combination of both CD4+ and CD8+ lymphocytes contributed to an early induction of disease, but also $\mathrm{CD} 4^{+}$lymphocytes alone. Although the underlying mechanism of $\mathrm{CD} 4+\mathrm{T}$-lymphocyte-mediated pathogenesis is yet unknown, one might speculate that MHC class II restricted cytotoxicity to virus-infected astrocytes is involved. JHM virus can infect astroglia and upregulate class II antigens in vitro 8 and Borna virus infected astrocytes from Lewis rats are recognized by class II restricted $\mathrm{CD}^{+}{ }^{+}$T-lymphocytes. ${ }^{9}$ However, so far it is not excluded that more indirect effects may also account to CD4+ cell-mediated pathogenesis, such as $\gamma$ interferon secretion by $\mathrm{CD} 4+$ cells that bind to class II expressing microglia. Since microglia upregulate class II antigens in JHM-infected rats 10 and virus is present in subacutely diseased animals as long as 3 weeks past infection 4 this hypothesis is not too far fetched. Moreover, it was recently shown that $\gamma$ interferon causes release of superoxyd anions in microglia cells in vitro ${ }^{11}$ a process that could also contribute to tissue damage.

Presence of B-lymphocytes in the transferred cell population was generally associated with a markedly delayed onset of clinical symptoms (table 1, \#6-\#8). Low titers of infectious virus in the brain of animals that received B-lymphocytes verify our previously published hypothesis that intracerebral synthesis of JHM-specific antibodies limits spread of the virus by neutralization ${ }^{5}$. There is of course one exception to this rule, in the case where $\mathrm{CD} 4+$ cells had been depleted prior to transfer (table 1, \#7). This result was not surprising at all, as it is known that most B-cells cannot act in the absence of syngeneic CD4+ cells which play a key role in the induction phase of the humoral immune response. Most likely this slight reduction of virus in this experiment has to be explained by the fact that the CD8+/B-lymphocyte transfer was contaminated with roughly $5 \%$ of $\mathrm{CD} 4+$ cells (from initially $48 \%$ ) that could provide some help for B-lymphocytes.

This protective character of the humoral immunity is known from different animal models and from man. Shubin et al.,11 reported that a nylon wool adherent fraction of immunized spleen cells (consisting mostly of B-lymphocytes) is necessary to reduce virus in the CNS of JHM virus-infected mice and Wege et al., ${ }^{3}$ showed that the incidence of JHM virus-induced encephalitis is markedly reduced in suckling rats nursed by JHM 
virus-immunized dams. This is in line with findings of Perlman et al.,13 who reported a shift in the disease pattern of JHM virus-induced encephalitis in mice from an acute lethal course to a milder subacute disease if infected animals were protected by maternal antibodies. Furthermore, in Theiler's virus infected mice, higher numbers of anti-viral IgM secreting cells could be isolated from the spleen of resistant strains, compared to susceptible ones 14 . In this case the protective role of the humoral immune response could be confirmed by passive transfer of serum from infected resistant strains to susceptible mice resulting in a marked decrease in the number of infected cells within the spinal cord. Nevertheless, although onset of the disease was delayed in animals reconstituted with CD4+ T- and B-lymphocytes, severity of the symptoms increased dramatically on 9 dpi and some animals died. Since CD8+ cells were lacking in these animals we assume, that B-lymphocytes can restrict viral spread and neutralize extracellular virus by antibody secretion but are unable to eliminate virus from tissue. Persistent viral antigen presentation in the brain could then provoke CD4+ cells to interact with class II expressing cells, resulting in tissue damage as already discussed above.

The disease-limiting effects of B-lymphocytes in the CNS tissue might be mainly explained by neutralization of infectious virus. However, another important consequence of virus-specific antibody secretion in the brain has to be discussed. High levels of antibodies may also strongly influence the histopathological changes in the JHM-infected brain and thus determine the clinically visible disease. Zimprich et al., 15 showed in the CNS of JHM virus infected LEW rats the presence of $\mathrm{IgG}+\mathrm{B}$-lymphocytes especially in demyelinated areas and an enhanced IgG content in macrophages within the plaques. Furthermore this group takes it as likely that the humoral response against the spike protein leads to a downregulation of this viral protein at least in neurons thus pushing the infection from gray to white matter. Such effects could contribute to the observed change in neurological disorders of our rats where white matter dysfunction (paresis or paralysis) was only observed in animals that received B-cells and CD4+ T-cells, whereas symptoms of gray matter

Table 1. Effect of lymphocyte transfer on the course of JHM virus infection in irradiated Lewis rats

\begin{tabular}{|c|c|c|c|c|c|c|c|c|}
\hline column ne & $\# 1$ & $\# 2$ & $\# 3$ & $\# 4$ & $\# 5$ & $\# 6$ & $\# 7$ & $\# 8$ \\
\hline irradiation & no & \multicolumn{7}{|c|}{$600 \mathrm{rad} \gamma$} \\
\hline \multirow{3}{*}{ cell transfer } & - & - & CD4+ & $a^{2}$ & $\mathrm{CD}_{4}{ }^{+}$ & $\mathrm{CD}^{+}+$ & $-*$ & $\mathrm{CD}^{+}+$ \\
\hline & - & - & - & CD8+ & CD8+ & - & CD8+ & CD8+ \\
\hline & - & - & - & - & - & B & B & B \\
\hline $\begin{array}{l}\text { onset of } \\
\text { disease }^{* *}\end{array}$ & 8 & 8 & 7 & 6 & 6 & 8 & 9 & 8 \\
\hline $\begin{array}{l}\text { maximal } \\
\text { severity }\end{array}$ & ++ & ++ & t++ & +++ & t+t & ++++ & ++ & ++ \\
\hline $\begin{array}{l}\text { virus recovery } \\
\text { at day } 9 \mathrm{pi}\end{array}$ & $<500$ & 8000 & 5100 & 9000 & 8000 & $8(0)$ & 46()$)$ & $<5())$ \\
\hline
\end{tabular}


disease were observed in the absence of the B-lymphocyte population.

In conclusion the presented data evidence, that a subclinical or clinically mild course of the intracerebral JHM infection is closely related to the presence of all lymphocyte subsets. Under the umbrella of antibody-secreting plasma cells viral spread is limited to few and small areas located mainly in the white matter. These sites are cleared from virus by the accompanying T-cell population. Absence of B-lymphocytes allows spread of virus to large areas of the CNS including the cerebral cortex and spinal cord and as a consequence severe clinical symptoms arise that are potentiated by a large number of inflammatory CD4+ and CD8+ T-lymphocytes 4 .

\section{ACKNOWLEDGEMENTS}

This study was supported by the Bundesministerium für Forschung und Technologie of the Fed. Rep. of Germany (Grant no.: KI 01 8839-2).

We are very grateful to Ursula Sauer and Marion Zips for their skillful technical assistance.

\section{REFERENCES}

1 F.S. Cheever, J.B. Daniels, A.M. Pappenheimer, \& O.T. Bailey, J. Exp. Med., 90:181 (1949)

2 K. Nagashima, H. Wege, R. Meyermann, \& V. ter Meulen, Acta Neuropath., (Berl), 44:63 (1978)

3 H. Wege, S. Siddell, \& V. ter Meulen, in: "Current Topics in Microbiology and Immunology", 99, M. Cooper, ed., Springer, (1982)

4 R. Dörries, S. Schwender, H. Imrich, \& H. Harms, Immunology, 74:539 (1991)

5 S. Schwender, H. Imrich \& R. Dörries, Immunology, 74:533 (1991)

6 N. Hirano, K. Fujiwara, S. Hino, \& N. Matsumoto, Arch. Ges. Virusforsch., 44:298 (1974)

7 J.O. Fleming, F.I. Wang, M.D. Trousdale, D.R. Hinton, \& S.A. Stohlman, in: "Coronaviruses and Their Diseases", D. Cavanagh and T.D.K. Brown, eds, Plenum Press, New York (199())

8 P.T. Massa, R. Dörries, \& V. ter Meulen, Nature, $320: 543$ (1986)

9 J.A. Richt, \& L. Stitz, Arch. Virol., 124:95 (1992)

10 J.D. Sedgwick, S. Schwender, R. Gregerson, R. Dörries, \& V. ter Meulen, J. Exp. Med., Submitted for publication Sept 1991

11 C.A. Colton, J. Yao, J.E. Keri, and D. Gilhert, J. Neuroimmunol., $30: 89$ (1992)

12 R.A. Shubin, M.A. Sussmann, J.O. Fleming, \& S.A. Stohlman, Microb. Pathog., 8:305 (1990)

13 S., Perlman, R. Schelper, E. Bolger, \& D. Ries, Microb. Pathog 2:185 (1987)

14 C.P. Rossi, E. Cash, C. Aubert, and A. Coutinho, J. Virol., 65:3895 (1991)

15 F. Zimprich, J. Winter, H. Wege, H. Lassmann, Neuropathol. Appl. Neurobiol. 17:469 (1991) 